detection. Results from our flow cytometry studies demonstrate that GD2/3 expression is significantly higher than EpCAM expression, across all OS cell lines within our panel. The cell capture efficiency strongly correlates with the cell surface expression data obtained from flow cytometry analysis. DISCUSSION/SIGNIFICANCE OF IMPACT: OS is the most common primary bone tumor and the third leading cause of pediatric cancer deaths. At diagnosis, $80 \%$ of patients will present with metastasis, however only $20 \%$ of these cases are clinically detectable. Innovative strategies to identify patients at risk of metastasis would allow for stratification of intervention therapies. Liquid biopsies are a novel alternative to current diagnostic imaging systems to monitor metastatic incidence and treatment efficacy. The detection of circulating tumor cells (CTCs) through routine blood sampling has the potential to be used clinically for earlier detection, monitoring the treatment of metastatic cancers and surveying the effect of therapeutic interventions on metastasis. To date, the majority of the studies on CTCs have evaluated their presence in carcinomas. Although sarcomas are rare, they generally have a poorer prognosis. This study will address one of the unmet medical needs in the field of CTC detection; the identification of cell surface OS makers to improve binding specificity, increase purity, and maintain a high capture efficiency.

3222

\section{University of Mississippi Center for Clinical and Translational Science (CCTS): A Catalyst for Clinical and Translational Sciences}

Leigh Ann Ross ${ }^{1}$, Christian R. Gomez ${ }^{1}$, Ingrid C. Espinoza ${ }^{1}$, Kim G. Adcock ${ }^{1}$ and Lauren S. Bloodworth ${ }^{1}$

${ }^{1}$ University of Mississippi

OBJECTIVES/SPECIFIC AIMS: To introduce CCTS to the clinical and translational research community. METHODS/STUDY POPULATION: Established in the summer of 2017, the Center for Clinical and Translational Science (CCTS) fosters cooperative clinical and translational sciences between the University of Mississippi School of Pharmacy (UMSOP) and the University of Mississippi Medical Center (UMMC). CCTS facilitates the translation of basic research discoveries into clinically validated therapies to improve the health of populations in Mississippi and beyond. Priority areas of investigation in CCTS include Cardiometabolic disorders, Cancer, Neuroscience, Infectious diseases, Precision Medicine, and Community-Based Research. To accomplish CCTS mission three overarching goals have been defined: I) Develop progressive and sustainable capacity for clinical and translational research in Mississippi; II) Promote interprofessional engagement in clinical and translational science; and III) Foster research collaboration among stakeholders in and outside of Mississippi. RESULTS/ANTICIPATED RESULTS: To carry its CCTS's mission three research units have been established: 1) The Pre-clinical Research Unit: Develops processes to move basic science discoveries towards translation into research in humans. This unit provides guidance in the development of Investigational New Drug (IND) applications; and identifies and pursues opportunities to develop progressive capacities for in vitro, ex vivo, in vivo, and in silico approaches for evaluating new pharmaceutical and therapeutic agents. 2) The Clinical Research Unit: Transitions projects that have received IND approval into the first phase of clinical trials. It also transitions clinical trials from Phase I to Phase II and to Phase III; develops standard operating procedures (SOPs), personnel training plans, and policies to guide clinical research; works with industry sponsors and governmental funding agencies; and assures compliance with regulatory requirements. 3) Community/population Research Unit: Develops, coordinates, and facilitates research activities and translation between clinical and community/population research stages. To do so, this unit works closely with community partners and Population Health programs on the Oxford and Jackson campuses. DISCUSSION/SIGNIFICANCE OF IMPACT: Since its inception, the CCTS has surpassed 1.5 million dollars in competitive funding. This early success positions the CCTS well to promote research collaboration between UMSOP and UMMC and to progress in becoming a national leader in clinical and translational investigation.

\section{6}

Who's ready to collaborate? Evaluating new measures of collaboration readiness among early career scholars in the CTSA network

Larry Hawk ${ }^{1}$, Eugene Maguin ${ }^{1}$, Timothy Murphy ${ }^{1}$,

Katherine Hartmann, MD, $\mathrm{PhD}^{2}$ and Morgan Jusko ${ }^{1}$

${ }^{1}$ University at Buffalo and ${ }^{2}$ Vanderbilt University Medical Center

OBJECTIVES/SPECIFIC AIMS: Many CTSA network activities aim to promote collaboration. Who should we target, and how should we evaluate short-term success? This study examined the validity of recently developed collaboration readiness indices among early career scholars, an important and understudied portion of the translational workforce. METHODS/STUDY POPULATION: Participants were 107 scholars within 10 years of completing terminal degree or residency (mean age $=38 ; 69 \%$ female; $29 \% \mathrm{MD}$ ) who applied to one of two week-long NCATS-funded Innovation Labs (www.buffalo.edu/innovationlabs.html). Measures included the MATRICx (Mallinson et al., 2016), which assesses 17 collaboration motivators and 31 threats; the Transdisciplinary Orientation Scale (TDO; Misra et al., 2015), an assessment of attitudes and behaviors theorized to predict effective collaboration; and a brief measure of one's perceived ability to succeed in different aspects of collaboration (i.e., self-efficacy; see teamscience.net). RESULTS/ANTICIPATED RESULTS: Factor analyses of individual measures and evaluation of cross-scale correlations suggest that collaboration readiness is multi-dimensional. Factor analysis of the MATRICx suggests 3 moderately-correlated facets of motivators (benefits to world, self, and others $\mathrm{rs}=+.50$ to +.62 ) and threats (process concerns, external barriers, and leadership style, $\mathrm{rs}=+.29$ to +.53 ). Most correlations between motivator and threat scales (except process concerns) were modest, suggesting they reflect relatively independent aspects of collaboration readiness. The TDO scales seemed to capture a different aspect of collaboration readiness; correlations with MATRICx motivator and threat scales were mostly modest ( $\mathrm{rs}=-.26$ to +.43 ). As expected, collaboration self-efficacy was positively related to collaboration motivators and TDO ( $\mathrm{rs}=+.41$ to +.59$)$ and negatively related to collaboration threats (particularly process threats, $r=-.47$ ). Participants typically scored in the upper half of the TDO, MATRICx motivator, and collaboration self-efficacy scale ranges, and in the lower half of the MATRICx threat scale ranges. DISCUSSION/SIGNIFICANCE OF IMPACT: Collaboration readiness is a reasonable short-term target of efforts to promote collaboration. However, this work suggests that no single scale captures the entire conceptual space, and multiple measures should be assessed. 
The implications for efforts to enhance collaboration are intriguing. In samples already high in collaboration readiness, these measures will have limited ability to detect positive change. However, assessment of collaboration readiness may be particularly useful in identifying scholars who could most benefit from collaborationenhancement programs (i.e., scholars with moderate scores on one or more of these metrics) and in personalizing intervention (e.g., selectively targeting TDO, collaboration motivators, and/or collaboration self-efficacy, and/or perceived threats to collaboration).

\section{Translational Science, Policy, \& Health Outcomes Science}

\author{
"My research is their business, but I'm not their \\ business": Patient and Oncologist Perspectives on the \\ Commercialization of Research Data \\ Kayte Spector-Bagdady ${ }^{1}$, Chris Krenz ${ }^{1}$, Michele Gornick ${ }^{1}$, \\ Collin Brummel ${ }^{1}$, Madison Kent ${ }^{1}$, J. Chad Brenner ${ }^{1}$, J. Scott Roberts ${ }^{1}$ \\ and Andrew G. Shuman ${ }^{1}$ \\ ${ }^{1}$ University of Michigan
}

OBJECTIVES/SPECIFIC AIMS: Our objective was to assess and compare the attitudes of patients with head and neck cancer and their clinicians regarding the commercialization of genetic research data. We explored whether such opinions changed when profits from such transactions were used to fund 1) cancer research, 2) academic research generally, or 3) if patients were given personalized genetic information in return. METHODS/STUDY POPULATION: This qualitative analysis was nested within a prospective precision oncology genomic sequencing study in an NCI-designated cancer center. We conducted paired, semi-structured interviews with enrolled participants with head \& neck cancer and with their doctors (medical oncologists, surgical oncologists, and radiation oncologists). Interviews were recorded, transcribed, and coded for analysis. Codes were developed through an iterative process until saturation was reached, and all transcripts were double-coded (and discrepancies reconciled) to ensure reliability. RESULTS/ANTICIPATED RESULTS: We identified three main themes from the patients and clinicians: (1) Both clinicians and their patients were unclear about how the study protocol and informed consent form authorized patients' genetic data to be used and commercialized in the future. (2) Patients with cancer were generally more comfortable than their clinician thought they were regarding the ongoing research use of their genetic data and commercialization thereof. (3) There is a strong interest among patients and clinicians in focusing academic medical center profits from commercialization back into the research program from which the data was acquired, rather than being invested into academic research more broadly. DISCUSSION/SIGNIFICANCE OF IMPACT: Given patients' strong feelings about the commercialization of their data, our results highlight the need for greater transparency-both with patients and with their clinicians - about potential future use of research data. Clinicians appear inclined to be particularly cautious regarding access to and commercialization of patients' data, however patients generally hope that their data may be used to help future cancer patients. Explicit discussions with patients about specific future uses of profits derived from commercialization of research data can ensure both transparency and participation in future primary and secondary precision health research programs.

3095

\section{Perceived Knowledge of Palliative Care among Immigrants: A Secondary Data Analysis from the Health Information National Trends Survey}

Amelia Barwise $^{1}$, Andrea Cheville ${ }^{2}$, Mark Wieland ${ }^{2}$, Ognjen Gajic ${ }^{2}$ and Alexandra Greenberg-Worisek ${ }^{2}$

${ }^{1}$ Mayo Clinic and ${ }^{2}$ Mayo Clinic Rochester,MN

OBJECTIVES/SPECIFIC AIMS: Immigrants to North America receive more interventions at end of life.The reasons for this are not entirely clear but may potentially be due to knowledge gaps. The primary objective of this study was to measure and describe levels of perceived knowledge about palliative care among immigrants to the US compared to those born in the US. Our secondary objective was to identify trusted sources for seeking information about palliative care among immigrants and compare these trusted sources with those born in the US. We hypothesized that immigrants would have less knowledge of palliative care than those born in US and would trust different sources for information about palliative care. METHODS/STUDY POPULATION: We analyzed data from the nationally representative 2018 Health Information National Trends Survey (HINTS 5, cycle 2). Questionnaires were administered via mail between January and May 2018 to a population-based sample of adults. The primary outcome of interest was assessed using the item "How would you describe your level of knowledge about palliative care?" The secondary outcome of interest was determined using the item "Imagine you had a strong need to get information about palliative care, which of the following would you most trust as a source of information about palliative care?" All analyses were complete case analyses and conducted with survey commands using SAS 9.3 (SAS Institute Inc, Cary, NC, USA). Descriptive statistics were calculated, and bivariate analyses run between the outcomes of interest and sociodemographic characteristics (age, sex, education, race/ ethnicity, nativity, English language proficiency). Multivariable logistic regressions were conducted to assess the role of nativity, controlling for statistically significant and relevant sociodemographic variables. Jackknife weighting was used to generate population-level estimates. RESULTS/ANTICIPATED RESULTS: The response rate was $33 \%(n=3384)$ and included $2846(85.3 \%$ weighted $)$ born in the US and 492 (14.7\% weighted) not born in the USA. About $70 \%$ of those born in the US and $77 \%$ of immigrants (weighted) responded that they had "never heard of palliative care." Trusted sources of palliative care were very similar between the groups (all $\mathrm{p}>0.05$ ). Both groups' preferred trusted source of palliative care knowledge was "health care provider," with over $80 \%$ of respondents in each group selecting this option. Printed materials and social media were the least popular trusted sources among both groups. After adjusting for relevant sociodemographic characteristics, we found no association between poor knowledge of palliative care and nativity $(\mathrm{p}=0.22)$. Female respondents had 2.5 -fold increased odds of reporting low levels of perceived knowledge of palliative care $(\mathrm{OR}=2.58$, $95 \% \mathrm{CI}=1.76-3.78 ; \mathrm{p}<0.001)$. Education was an important predictor of perceived knowledge of palliative care; as education level increased, so did perceived knowledge of palliative care $(\mathrm{p}<0.001)$. DISCUSSION/SIGNIFICANCE OF IMPACT: Perceived knowledge of palliative care is poor generally, regardless of birthplace. Trusted 